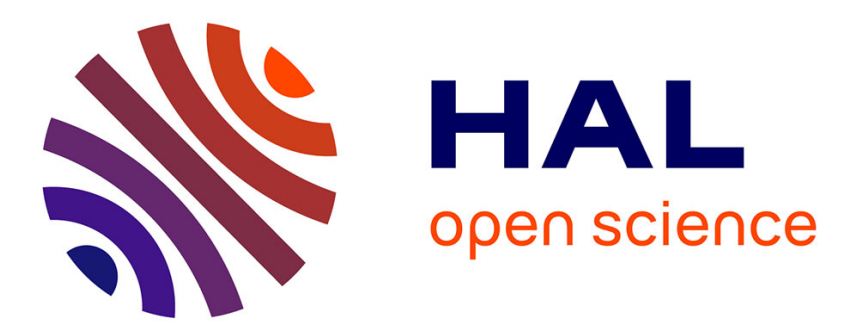

\title{
Compositionality and Generalization in Emergent Languages
}

Rahma Chaabouni, Eugene Kharitonov, Diane Bouchacourt, Emmanuel Dupoux, Marco Baroni

\section{- To cite this version:}

Rahma Chaabouni, Eugene Kharitonov, Diane Bouchacourt, Emmanuel Dupoux, Marco Baroni. Compositionality and Generalization in Emergent Languages. ACL 2020 - 8th annual meeting of the Association for Computational Linguistics, Jul 2020, Seattle / Virtual, United States. hal-02959466

\section{HAL Id: hal-02959466 https://hal.science/hal-02959466}

Submitted on 6 Oct 2020

HAL is a multi-disciplinary open access archive for the deposit and dissemination of scientific research documents, whether they are published or not. The documents may come from teaching and research institutions in France or abroad, or from public or private research centers.
L'archive ouverte pluridisciplinaire $\mathbf{H A L}$, est destinée au dépôt et à la diffusion de documents scientifiques de niveau recherche, publiés ou non, émanant des établissements d'enseignement et de recherche français ou étrangers, des laboratoires publics ou privés. 


\title{
Compositionality and Generalization in Emergent Languages
}

\author{
Rahma Chaabouni ${ }^{1,2 *}$, Eugene Kharitonov ${ }^{1 *}$, Diane Bouchacourt ${ }^{1}$, Emmanuel Dupoux $^{1,2}$, and Marco Baroni ${ }^{1,3}$ \\ ${ }^{1}$ Facebook AI Research \\ ${ }^{2}$ Cognitive Machine Learning (ENS - EHESS - PSL Research University - CNRS - INRIA) \\ ${ }^{3}$ ICREA \\ \{rchaabouni, kharitonov, dianeb, dpx, mbaroni\}@efb.com
}

\begin{abstract}
Natural language allows us to refer to novel composite concepts by combining expressions denoting their parts according to systematic rules, a property known as compositionality. In this paper, we study whether the language emerging in deep multi-agent simulations possesses a similar ability to refer to novel primitive combinations, and whether it accomplishes this feat by strategies akin to human-language compositionality. Equipped with new ways to measure compositionality in emergent languages inspired by disentanglement in representation learning, we establish three main results. First, given sufficiently large input spaces, the emergent language will naturally develop the ability to refer to novel composite concepts. Second, there is no correlation between the degree of compositionality of an emergent language and its ability to generalize. Third, while compositionality is not necessary for generalization, it provides an advantage in terms of language transmission: The more compositional a language is, the more easily it will be picked up by new learners, even when the latter differ in architecture from the original agents. We conclude that compositionality does not arise from simple generalization pressure, but if an emergent language does chance upon it, it will be more likely to survive and thrive.
\end{abstract}

\section{Introduction}

Most concepts we need to express are composite in some way. Language gives us the prodigious ability to assemble messages referring to novel composite concepts by systematically combining expressions denoting their parts. As interest raises in developing deep neural agents evolving a communication code to better accomplish cooperative tasks, the question arises of how the emergent code can be

\footnotetext{
${ }^{*}$ Contributed equally.
}

endowed with the same desirable compositionality property (Kottur et al., 2017; Lazaridou et al., 2018; Mordatch and Abbeel, 2018; Cogswell et al., 2019; $\mathrm{Li}$ and Bowling, 2019). This in turn requires measures of how compositional an emergent language is (Andreas, 2019). Compositionality is a core notion in linguistics (Partee, 2004), but linguists' definitions assume full knowledge of primitive expressions and their combination rules, which we lack when analyzing emergent languages (Nefdt, 2020). Also, these definitions are categorical, whereas to compare emergent languages we need to quantify degrees of compositionality.

Some researchers equate compositionality with the ability to correctly refer to unseen composite inputs (e.g., Kottur et al., 2017; Cogswell et al., 2019). This approach measures the generalization ability of a language, but it does not provide any insights on how this ability comes about. Indeed, one of our main results below is that emergent languages can attain perfect generalization without abiding to intuitive notions of compositionality.

Topographic similarity has become the standard way to quantify the compositionality of emergent languages (e.g., Brighton and Kirby, 2006; Lazaridou et al., 2018; Li and Bowling, 2019). This metric measures whether the distance between two meanings correlates with the distance between the messages expressing them. While more informative than generalization, topographic similarity is still rather agnostic about the nature of composition. For example, when using, as is standard practice, Levenshtein distance to measure message distance, an emergent language transparently concatenating symbols in a fixed order and one mixing deletion and insertion operations on free-ordered symbols can have the same topographic similarity.

We introduce here two more "opinionated" measures of compositionality that capture some intuitive properties of what we would expect to hap- 
pen in a compositional emergent language. One possibility we consider is that order-independent juxtapositions of primitive forms could denote the corresponding union of meanings, as in English noun conjunctions: cats and $\operatorname{dog} s, \operatorname{dog} s$ and cats. The second still relies on juxtaposition, but exploits order to denote different classes of meanings, as in English adjective-noun phrases: red triangle, blue square. Both strategies result in disentangled messages, where each primitive symbol (or symbol+position pair) univocally refers to a distinct primitive meaning independently of context. We consequently take inspiration from work on disentanglement in representation learning (Suter et al., 2019) to craft measures that quantify whether an emergent language follows one of the proposed composition strategies.

Equipped with these metrics, we proceed to ask the following questions. First, are neural agents able to generalize to unseen input combinations in a simple communication game? We find that generalizing languages reliably emerge when the input domain is sufficiently large. This somewhat expected result is important nevertheless, as failureto-generalize claims in the recent literature are often based on very small input spaces. Second, we unveil a complex interplay between compositionality and generalization. On the one hand, there is no correlation between our compositionality metrics and the ability to generalize, as emergent languages successfully refer to novel composite concepts in inscrutablly entangled ways. (Order-dependent) compositionality, however, if not necessary, turns out to be a sufficient condition for generalization. Finally, more compositional languages are easier to learn for new agents, including agents that are architecturally different from the ones that evolved the language. This suggests that, while composition might not be a "natural" outcome of the need to generalize, it is a highly desirable one, as compositional languages will more easily be adopted by a large community of different agents. We return to the implications of our findings in the discussion.

\section{Setup}

\subsection{The game}

We designed a variant of Lewis' signaling game (Lewis, 1969). The game proceeds as follows:

1. Sender network receives one input $i$ and chooses a sequence of symbols from its vo- cabulary $V=\left\{s_{1}, s_{2} \ldots, s_{c_{v o c}}\right\}$ of size $c_{v o c}$ to construct a message $m$ of fixed length $c_{l e n}$.

2. Receiver network consumes $m$ and outputs $\hat{\boldsymbol{i}}$.

3. Agents are successful if $\hat{i}=i$, that is, Receiver reconstructs Sender's input.

Each input $i$ of the reconstruction game is comprised of $i_{\text {att }}$ attributes, each with $i_{\text {val }}$ possible values. We let $i_{\text {att }}$ range from 2 to 4 and $i_{\text {val }}$ from 4 to 100 . We represent each attribute as a $i_{v a l}$ onehot vector. An input $i$ is given by the concatenation of its attributes. For a given $\left(i_{a t t}, i_{v a l}\right)$, the number of input samples $|I|=i_{\text {val }}^{i_{\text {att }}}$.

This environment, which can be seen as an extension of that of Kottur et al. (2017), is one of the simplest possible settings to study the emergence of reference to composite concepts (here, combinations of multiple attributes). Attributes can be seen as describing object properties such as color and shape, with their values specifying those properties for particular objects (red, round). Alternatively, they could be seen as slots in an abstract semantic tree (e.g., agent and action), with the values specifying their fillers (e.g., dog, barking). In the name of maximally simplifying the setup and easing interpretability, unlike Kottur et al. (2017), we consider a single-step game. We moreover focus on input reconstruction instead of discrimination of a target input among distractors as the latter option adds furtherx complications: for example, languages in that setup have been shown to be sensitive to the number and distribution of the distractors (Lazaridou et al., 2018).

For a fixed $|I|$, we endow Sender with large enough channel capacity $|C|=c_{\text {voc }}^{c_{l e n}}\left(c_{\text {voc }} \in\right.$ $\{5,10,50,100\}$ and $\left.c_{l e n} \in\{3,4,6,8\}\right)$ to express the whole input space (i.e., $|C| \geq|I|$ ). Unless explicitly mentioned, we run 10 different initializations per setting. See Appendix 8.1 for details about the range of tested settings. The game is implemented in EGG (Kharitonov et al., 2019). ${ }^{1}$

\subsection{Agent architecture}

Both agents are implemented as single-layer GRU cells (Cho et al., 2014) with hidden states of size $500 .^{2}$ Sender encodes $i$ in a message $m$ of fixed

\footnotetext{
${ }^{1}$ Code can be found at https://github.com/ facebookresearch/EGG/tree/master/egg/ zoo/compo_vs_generalization.

${ }^{2}$ Experiments with GRUs of different capacity are reported in the Appendix. We also informally replicated our main
} 
length $c_{l e n}$ as follows. First, a linear layer maps the input vector into the initial hidden state of Sender. Next, the message is generated symbol-by-symbol by sampling from a Categorical distribution over the vocabulary $c_{v o c}$, parameterized by a linear mapping from Sender's hidden state. The generated symbols are fed back to the cell. At test time, instead of sampling, symbols are selected greedily.

Receiver consumes the entire message $m$. Further, we pass its hidden state through a linear layer and consider the resulting vector as a concatenation of $i_{\text {att }}$ probability vectors over $i_{\text {val }}$ values each. As a loss, we use the average cross-entropy between these distributions and Sender's input.

\subsection{Optimization}

Popular approaches for training with discrete communication include Gumbel-Softmax (Maddison et al., 2016; Jang et al., 2016), REINFORCE (Williams, 1992), and a hybrid in which the Receiver gradients are calculated via backpropagation and those of Sender via REINFORCE (Schulman et al., 2015). We use the latter, as recent work (e.g., Chaabouni et al., 2019) found it to converge more robustly. We apply standard tricks to improve convergence: (a) running mean baseline to reduce the variance of the gradient estimates (Williams, 1992), and (b) a term in the loss that favors higher entropy of Sender's output, thus promoting exploration. The obtained gradients are passed to the Adam optimizer (Kingma and $\mathrm{Ba}$, 2014) with learning rate 0.001 .

\section{Measurements}

\subsection{Compositionality}

Topographic similarity (topsim) (Brighton and Kirby, 2006) is commonly used in language emergence studies as a quantitative proxy for compositionality (e.g., Lazaridou et al., 2018; Li and Bowling, 2019). Given a distance function in the input space (in our case, attribute value overlap, as attributes are unordered, and values categorical) and a distance function in message space (in our case, following standard practice, minimum edit distance between messages), topsim is the (Spearman) correlation between pairwise input distances and the corresponding message distances. The measure can detect a tendency for messages with similar meanings to be similar in form, but it is relatively

results with LSTMs, that were slower to converge. We were unable to adapt Transformers to successfully play our game. agnostic about the type of similarity (as long as it is captured by minimum edit distance).

We complement topsim with two measures that probe for more specific types of compositionality, that we believe capture what deep-agent emergentlanguage researchers seek for, when interested in compositional languages. In most scenarios currently considered in this line of research, the composite inputs agents must refer to are sets or sequences of primitive elements: for example, the values of a set of attributes, as in our experiment. In this restricted setup, a compositional language is a language where symbols independently referring to primitive input elements can be juxtaposed to jointly refer to the input ensembles. Consider a language with a symbol $r$ referring to input element color:red and another symbol $l$ referring to weight:light, where $r$ and $l$ can be juxtaposed (possibly, in accordance with the syntactic rules of the language) to refer to the input set \{color:red, weight:light $\}$. This language is intuitively compositional. On the other hand, a language where both $r$ and $l$ refer to these two input elements, but only when used together, whereas other symbol combinations would refer to color:red and weight:light in other contexts, is intuitively not compositional. Natural languages support forms of compositionality beyond the simple juxtaposition of contextindependent symbols to denote ensembles of input elements we are considering here (e.g., constructions that denote the application of functions to arguments). However, we believe that the proposed intuition is adequate for the current state of affairs in language emergence research.

The view of compositionality we just sketched is closely related to the idea of disentanglement in representation learning. Disentangled representations are expected to enable a consequent model to generalize on new domains and tasks (Bengio et al., 2013). Even if this claim has been challenged (Bozkurt et al., 2019; Locatello et al., 2019), several interesting metrics have been proposed to quantify disentanglement, as reviewed in Suter et al. (2019). We build in particular upon the Information Gap disentanglement measure of Chen et al. (2018), evaluating how well representations capture independence in the input sets.

Our positional disentanglement (posdis) metric measures whether symbols in specific positions tend to univocally refer to the values of a specific attribute. This order-dependent strategy is com- 
monly encountered in natural language structures (and it is a pre-condition for sophisticated syntactic structures to emerge). Consider English adjectivenoun phrases with a fully intersective interpretation, such as yellow triangle. Here, the words in the first slot will refer to adjectival meanings, those in the second to nominal meanings. In our simple environment, it might be the case that the first symbol is used to discriminate among values of an attribute, and the second to discriminate among values of another attribute. Let's denote $s_{j}$ the $j_{-}$ th symbol of a message and $a_{1}^{j}$ the attribute that has the highest mutual information with $s_{j}: a_{1}^{j}=$ $\arg \max _{a} \mathcal{I}\left(s_{j} ; a\right)$. In turn, $a_{2}^{j}$ is the second highest informative attribute, $a_{2}^{j}=\arg \max _{a \neq a_{1}^{j}} \mathcal{I}\left(s_{j} ; a\right)$. Denoting $\mathcal{H}\left(s_{j}\right)$ the entropy of $j$-th position (used as a normalizing term), we define posdis as:

$$
\text { posdis }=1 / c_{\text {len }} \sum_{j=1}^{c_{\text {len }}} \frac{\mathcal{I}\left(s_{j} ; a_{1}^{j}\right)-\mathcal{I}\left(s_{j} ; a_{2}^{j}\right)}{\mathcal{H}\left(s_{j}\right)}
$$

We ignore positions with zero entropy. Eq. 1 captures the intuition that, for a language to be compositional given our inputs, each position of the message should only be informative about a single attribute. However, unlike the related measure proposed by Resnick et al. (2019), it does not require knowing which set of positions encodes a particular attribute, which makes it computationally simpler (only linear in $c_{l e n}$ ).

Posdis assumes that a language uses positional information to disambiguate symbols. However, we can easily imagine a language where symbols univocally refer to distinct input elements independently of where they occur, making order irrelevant. ${ }^{3}$ Hence, we also introduce bag-of-symbols disentanglement (bosdis). The latter maintains the requirement for symbols to univocally refer to distinct meanings, but captures the intuition of a permutation-invariant language, where only symbol counts are informative. Denoting by $n_{j}$ a counter of the $j$-th symbol in a message, bosdis is given by:

$$
\text { bosdis }=1 / c_{\text {voc }} \sum_{j=1}^{c_{\text {voc }}} \frac{\mathcal{I}\left(n_{j} ; a_{1}^{j}\right)-\mathcal{I}\left(n_{j} ; a_{2}^{j}\right)}{\mathcal{H}\left(n_{j}\right)}
$$

In all experiments, the proposed measures topsim, posdis and bosdis are calculated on the train set.

\footnotetext{
${ }^{3}$ This is not unlike what happens in order-insensitive constructions such as English conjunctions: dogs and cats, cats and dogs.
}

In Appendix 8.2, we illustrate how the three metrics behave differently on three miniature languages. Across the languages of all converging runs in our simulations, their Spearman correlations are: topsim/posdis: 0.08 ; topsim/bosdis: 0.38; posdis/bosdis: 0.31 . These correlations, while not extremely high, are statistically significant ( $p<0.01$ ), which is reassuring as all metrics attempt to capture compositionality. It is also in line with reasonable expectations that the most "opinionated" posdis measure is the one that behaves most differently from topsim.

\subsection{Generalization}

In our setup, generalization can be straightforwardly measured by splitting all possible distinct inputs so that the test set only contains inputs with attribute combinations that were not observed at training. Generalization is then simply quantified by test accuracy. In intuitive terms, at training time the agents are exposed to blue triangles and red circles, but blue circles only appear at test time. This requires Sender to generate new messages, and Receiver to correctly infer their meaning. If a blue circle is accurately reconstructed, then agents do generalize.

For all the considered settings, we split the possible distinct inputs into $90 \%$ train and $10 \%$ test items. This implies that the absolute training/test set sizes increase with input dimension (this issue is further discussed in Appendix 8.4).

Finally, we only evaluate generalization for runs that successfully converged, where convergence is operationalized as $>99.9 \%$ training-set accuracy.

\section{Generalization emerges "naturally" if the input space is large}

Fig. 1 shows that emergent languages are able to almost perfectly generalize to unseen combinations as long as input size $|I|$ is sufficiently large (input size/test accuracy Spearman $\rho=0.86, p \approx 0$ ). The figure also shows that the way in which a large input space is obtained (manipulating $i_{a t t}$ or $i_{v a l}$ ) does not matter (no significant accuracy difference between the bracketed runs, according to a set of t-tests with $p>0.01$ ). Moreover, the correlation is robust to varying agents' capacity (Appendix 8.3; see Resnick et al. (2019) for a thorough study of how agent capacity impacts generalization and compositionality). Importantly, the effect is not simply a product of larger input sizes coming with 


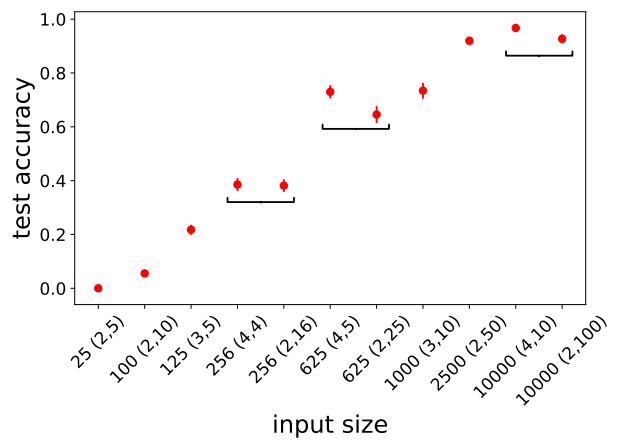

Figure 1: Average accuracy on unseen combinations as a function of input size of successful runs. The x-axis is ordered by increasing input size $|I|$. Brackets denote $\left(i_{a t t}, i_{v a l}\right)$. Vertical bars represent the standard error of the mean (SEM). Horizontal brackets group settings with same $|I|$ but different $\left(i_{a t t}, i_{v a l}\right)$.

larger training corpora, as we replicate it in Appendix 8.4 while keeping the number of distinct training examples fixed, but varying input combinatorial variety. What matters is that, in the training data, specific attribute values tend to occur with a large range of values from other attributes, providing a cue about the composite nature of the input.

That languages capable to generalize will only emerge when the input is varied enough might seem obvious, and it has been shown before in mathematical simulations of language emergence (Nowak et al., 2000), as well as in studies of deep network inductive biases (Zhao et al., 2018). However, our result suggests an important caveat when interpreting experiments based on small input environments that report failures in the generalization abilities of deep networks (e.g., Kottur et al., 2017; Lake and Baroni, 2018). Before assuming that special architectures or training methods are needed for generalization to emerge, such experiments should be repeated with much larger/varied input spaces, where it is harder for agents to develop ad-hoc strategies overfitting the training data and failing to generalize.

We also considered the relation between channel capacity $|C|$ and language emergence. Note that $|C| \geq|I|$ is a prerequisite for successful communication, and a perfectly compositional language could already generalize at the lower $|C|=|I|$ bound. Indeed, limiting channel capacity has been proposed as an important constraint for the emergence of compositionality (Nowak and Krakauer, 1999). However, we find that, when $|I|$ is sufficiently large to support generalization, our deep agents need $|C|>|I|$ in order to even converge at training time. The minimum $|C| /|I|$ ratio across all converging runs for each configuration with $|I| \geq 625$ (the settings where we witness generalizing languages) is on average 5.9 (s.d.: 4.4).

Concretely, this implies that none of our successful languages is as compact as a minimal fullycompositional solution would afford. Appendix 8.5 reports experiments focusing, more specifically, on the relation between channel capacity and generalization, showing that it is essential for $|C|$ to be above a large threshold to reach near-perfect accuracy, and further increasing $|C|$ beyond that does not hamper generalization.

\section{Generalization does not require compositionality}

Having established that emergent languages can generalize to new composite concepts, we test whether languages that generalize better are also more compositional. Since bosdis and topsim correlate with $|C|$ (Appendix 8.6), we compute Spearman correlations between test accuracy and compositionality metrics across all converging runs of each $\left(i_{a t t}, i_{v a l}, c_{l e n}, c_{v o c}\right)$ configuration separately. Surprisingly, in just 4 out of 141 distinct settings the correlation is significant $(p<0.01)$ for at least 1 measure. $^{4}$

We further analyze the $\left(i_{\text {att }}=2, i_{\text {val }}=100, c_{\text {len }}=3\right.$, $\left.c_{v o c}=100\right)$ setting, as it has a large number of generalizing runs, and it is representative of the general absence of correlation we also observe elsewhere. Fig. 2 confirms that even non-compositional languages (w.r.t. any definition of compositionality) can generalize well. Indeed, for very high test accuracy $(>98 \%)$, we witness a large spread of posdis (between 0.02 and 0.72), bosdis (between 0.03 and 0.4) and topsim (between 0.11 and 0.64). In other words, deep agents are able to communicate about new attribute combinations while using noncompositional languages. We note moreover that even the most compositional languages according to any metric are far from the theoretical maximum (=1 for all metrics).

We observe however that the top-left quadrants of Fig. 2 panels are empty. In other words, it never happens that a highly compositional language has low accuracy. To verify this more thoroughly, for each compositionality measure $\mu$, we select those languages, among all converging runs in all con-

\footnotetext{
${ }^{4} 3,3$ and 1 (different) significant settings for topsim, posdis and bosdis, respectively.
} 
figurations, that have $\mu>0.5$, and compute the proportion of them that reaches high test accuracy $(>0.80)$. We find that this ratio equates $0.90,0.50$, and 0.11 for posdis, bosdis, and topsim respectively. That is, while compositionality is not a necessary condition for generalization, it appears that the strongest form of compositionality, namely posdis, is at least sufficient for generalization. This provides some evidence that compositionality is still a desirable feature, as further discussed in Section 6 .

We gain further insights on what it means to generalize without full compositionality by taking a deeper look at the language shown in red in Fig. 2, that has near-perfect generalization accuracy (>99\%), and whose posdis score (0.70), while near the relative best, is still far from the theoretical maximum (we focus on posdis since it is the easiest compositional strategy to qualitatively characterize). As its behavior is partially interpretable, this "medium-posdis" language offered us clearer insights than more strongly entangled cases. We partially analyze one of the latter in Appendix 8.7.

Note that, with $\left(i_{a t t}=2, i_{\text {val }}=100\right)$, a $\left(c_{l e n}=2\right.$, $\left.c_{v o c}=100\right)$ channel should suffice for a perfectly positionally disentangled strategy. Why does the analyzed language use $\left(c_{l e n}=3\right)$ instead? Looking at its mutual information profile (Appendix Table 5), we observe that positions 2 and 3 (pos 2 and pos 3$)$ are respectively denoting attributes 2 and 1 (att2 and att1): pos 3 has high mutual information with att 1 and low mutual information with att2; the opposite holds for pos 2 . The remaining position, pos 1 , could then be simply redundant with respect to the others, or encode noise ignored by Receiver. However, this is not quite the case, as the language settled instead for a form of "leaky disentanglement". The two disentangled positions do most of the job, but the third, more entangled one, is still necessary for perfect communication.

To see this, consider the ablations in Table 1 . Look first at the top block, where the trained Receiver of the relevant run is fed messages with the symbol in one original position preserved, the others shuffled. Confirming that communication is largely happening by disentangled means, preserving pos 2 alone suffices to have Receiver guessing a large majority of att 2 values, and keeping pos3 unchanged is enough to guess almost $90 \%$ of attl values correctly. Conversely, preserving pos 1 alone causes a complete drop in accuracy for both attributes. However, neither pos 2 nor pos 3 are sufficient on their own to perfectly predict the corresponding attributes. Indeed, the results in the bottom block of the table (one symbol shuffled while the others stay in their original position) confirm that pos 1 carries useful complementary information: when fixing the latter and either one of the other positions, we achieve $100 \%$ accuracy for the relevant attribute (att 2 for $p o s 1+p o s 2$ and att 1 for pos $1+$ pos3), respectively.

In sum, pos 2 and pos 3 largely specialized as predictors of att 2 and att 1 , respectively. However, they both have a margin of ambiguity (in pos 2 and pos 3 there are 96 and 98 symbols effectively used, respectively, whereas a perfect 1-to-1 strategy would require 100). When the symbols in these positions do not suffice, pos 1 , that can refer to both attributes, serves a disambiguating role. We quantified this complementary function as follows. We define the cue validity of $s_{p}$ (symbol in position $p$ ) w.r.t an attribute $a$ as $C V\left(s_{p}, a\right)=\max _{\bar{a}} P\left(\bar{a} \mid s_{p}\right)$, where $\bar{a}$ iterates over all possible values of $a$. $C V\left(s_{\text {pos } 1}, a t t 2\right)$ is significantly higher in those (train/test) messages where $C V\left(s_{\text {pos } 2}\right.$, att 2$)$ is below average. Similarly, $C V\left(s_{\text {pos } 1}, a t t 1\right)$ is significantly higher in messages where $C V\left(s_{\text {pos } 3}, a t t 1\right)$ is below average ( $p \approx 0$ in both cases). We might add that, while there is a huge difference between our simple emergent codes and natural languages, the latter are not perfectly disentangled either, as they feature extensive lexical ambiguity, typically resolved in a phrasal context (Piantadosi et al., 2012).

\begin{tabular}{ll|r|r|r} 
& & att & att2 & both atts \\
\hline fixing & posl & 1 & 3 & 0 \\
1 position & pos 2 & 1 & 68 & 0 \\
& pos3 & 89 & 1 & 1 \\
\hline shuffling & pos 1 & 89 & 69 & 61 \\
1 position & pos 2 & 100 & 3 & 3 \\
& pos3 & 1 & 100 & 1
\end{tabular}

Table 1: Feeding shuffled messages from the analyzed language to the corresponding trained Receiver. Average percentage accuracy across 10 random shufflings (s.d. always $\approx 0$ ) when: top: symbols in all positions but one are shuffled across the data-set; bottom: symbols in a single position are shuffled across the data-set. The data-set includes all training and test messages produced by the trained Sender and correctly decoded in their original form by Receiver ( $>99 \%$ of total messages). 


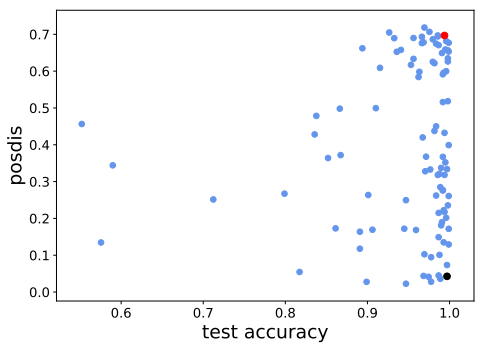

(a) posdis

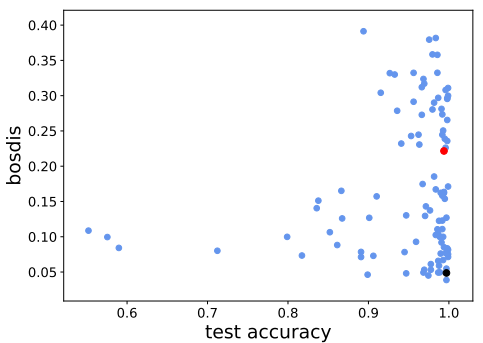

(b) bosdis

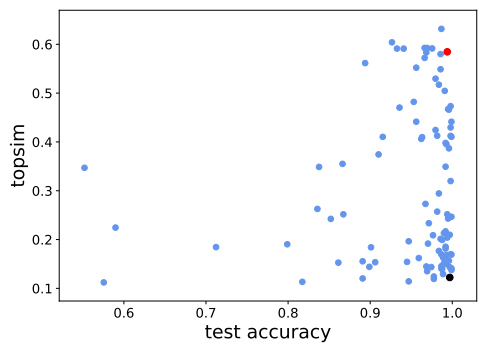

(c) topsim

Figure 2: Compositionality in function of generalization. Each point represents a successful run in the $\left(i_{a t t}=2\right.$, $\left.i_{v a l}=100, c_{l e n}=3, c_{v o c}=100\right)$ setting. Red and black points correspond respectively to the medium- and lowdisentanglement languages analyzed in Section 5 and Appendix 8.7.

\section{Compositionality and ease of transmission}

The need to generalize to new composite inputs does not appear to constitute a sufficient pressure to develop a compositional language. Given that compositionality is ubiquitous in natural language, we conjecture that it has other beneficial properties, making it advantageous once agents chanced upon it. Compositional codes are certainly easier to read out by humans (as shown by our own difficulty in qualitatively analyzing highly entangled languages), and we might hypothesize that this ease-of-decoding is shared by computational agents. A long tradition of subject studies and computational simulations has shown that the need to transmit a language across multiple generations or to populations of new learners results in the language being more compositional (e.g., Kirby, 2001; Kirby et al., 2015; Verhoef et al., 2016; Cornish et al., 2017; Cogswell et al., 2019; Guo et al., 2019; $\mathrm{Li}$ and Bowling, 2019). Our next experiments are closely related to this earlier work, but we adopt the opposite perspective. Instead of asking whether the pressure to transmit a language will make it more compositional, we test whether languages that have already emerged as compositional, being easier to decode, are more readily transmitted to new learners. ${ }^{5}$

Specifically, we run 30 games in the largest input setting $\left(i_{a t t}=2, i_{v a l}=100\right)$, varying the channel parameters. We select the pairs of agents that achieved a high level of generalization accuracy $(\geq 0.80)$. Next, following the paradigm of $\mathrm{Li}$ and Bowling (2019), we freeze Sender, and train a new

\footnotetext{
${ }^{5} \mathrm{Li}$ and Bowling (2019) established this for hand-crafted languages; we extend the result to spontaneously emerging ones.
}

Receiver from scratch. We repeat this process 3 times per game, initializing new Receivers with different random seeds. Once the newly formed pair of agents is successful on the training set, we measure its test accuracy. We also report speed of learning, measured by area under the epochs vs. training accuracy curve. We experiment with three Receiver architectures. The first two, GRU (500) and GRU (50), are GRUs with hidden layer sizes of 500 (identical to the original Receiver) and 50, respectively. The third is a two-layer Feed-Forward Network (FFN) with a ReLu non-linearity and hidden size 500. The latter Receiver takes the flattened one-hot representation of the message as its input. This setup allows probing ease of language transmission across models of different complexity. We leave the study of language propagation across multiple generations of speakers to future work.

Results in the same setting studied in Section 5 are presented in Table 2 (experiments with other setups are in Appendix 8.8). Both learning speed and generalization accuracy of new Receivers are strongly positively correlated with degree of compositionality. The observed correlations reach values almost as high as 0.90 for learning speed and 0.80 for generalization, supporting our hypothesis that, when emergent languages are compositional, they are simpler to understand for new agents, including smaller ones (GRU (50)), and those with a different architecture (FFN).

\section{Discussion}

The natural emergence of generalization There has been much discussion on the generalization capabilities of neural networks, particularly in linguistic tasks where humans rely on compositionality (e.g., Fodor and Lepore, 2002; Marcus, 


\begin{tabular}{|c|c|c|c|c|c|c|c|c|c|}
\hline & \multicolumn{3}{|c|}{ posdis } & \multicolumn{3}{|c|}{ bosdis } & \multicolumn{3}{|c|}{ topsim } \\
\hline & GRU(500) & GRU(50) & FFN & GRU(500) & GRU(50) & FFN & GRU(500) & GRU(50) & FFN \\
\hline Learning Speed & 0.87 & 0.71 & 0.35 & 0.85 & 0.68 & 0.33 & 0.87 & 0.71 & 0.35 \\
\hline Generalization & 0.80 & 0.55 & 0.50 & 0.81 & 0.55 & 0.51 & 0.79 & 0.54 & 0.48 \\
\hline
\end{tabular}

Table 2: Spearman correlation between compositionality metrics and ease-of-transmission measures for $\left(i_{a t t}=2\right.$, $\left.i_{v a l}=100, c_{l e n}=3, c_{v o c}=100\right)$. All values are statistically significant $(p<0.01)$.

2003; van der Velde et al., 2004; Brakel and Frank, 2009; Kottur et al., 2017; Lake and Baroni, 2018; Andreas, 2019; Hupkes et al., 2019; Resnick et al., 2019). In our setting, the emergence of generalization is very strongly correlated with variety of the input environment. While this result should be replicated in different conditions, it suggests that it is dangerous to study the generalization abilities of neural networks in "thought experiment" setups where they are only exposed to a small pool of carefully-crafted examples. Before concluding that garden-variety neural networks do not generalize, the simple strategy of exposing them to a richer input should always be tried. Indeed, even studies of the origin of human language conjecture that the latter did not develop sophisticated generalization mechanisms until pressures from an increasingly complex environment forced it to evolve in that direction (Bickerton, 2014; Hurford, 2014).

Generalization without compositionality Our most important result is that there is virtually no correlation between whether emergent languages are able to generalize to novel composite inputs and the presence of compositionality in their messages (Andreas (2019) noted in passing the emergence of non-compositional generalizing languages, but did not explore this phenomenon systematically). Supporting generalization to new composite inputs is seen as one of the core purposes of compositionality in natural language (e.g., Pagin and Westerståhl, 2010). While there is no doubt that compositional languages do support generalization, we also found other systems spontaneously arising that generalize without being compositional, at least according to our intuitive measures of compositionality. This has implications for the ongoing debate on the origins of compositionality in natural language, (e.g., Townsend et al., 2018, and references there), as it suggests that the need to generalize alone might not constitute a sufficient pressure to develop a fully compositional language. Our result might also speak to those linguists who are exploring the non-fully-compositional corners of natural language (e.g., Goldberg, 2019). A thorough investigation of neural network codes that can generalize while being partially entangled might shed light on similar phenomena in human languages. Finally, and perhaps most importantly, recent interest in compositionality among AI researchers stems from the assumption that compositionality is crucial to achieve good generalization through language (e.g., Lake and Baroni, 2018; Lazaridou et al., 2018; Baan et al., 2019). Our results suggest that the pursuit of generalization might be separated from that of compositionality, a point also recently made by Kharitonov and Baroni (2020) through hand-crafted simulations.

What is compositionality good for? We observed that positional disentanglement, while not necessary, is sufficient for generalization. If agents develop a compositional language, they are then very likely to be able to use it correctly to refer to novel inputs. This supports the intuition that compositional languages are easier to fully understand. Indeed, when training new agents on emerged languages that generalize, it is much more likely that the new agents will learn them fast and thoroughly (i.e., they will be able to understand expressions referring to novel inputs) if the languages are already compositional according to our measures. That language transmission increases pressure for structured representations is an established fact (e.g., Kirby et al., 2015; Cornish et al., 2017). Here, we reversed the arrow of causality and showed that, if compositionality emerges (due to chance during initial language development), it will make a language easier to transmit to new agents. Compositionality might act like a "dominant" genetic feature: it might arise by a random mutation but, once present, it will survive and thrive, as it guarantees that languages possessing it will generalize and will be easier to learn. From an AI perspective, this suggests that trying to enforce compositionality during language emergence will increase the odds 
of developing languages that are quickly usable by wide communities of artificial agents, that might be endowed with different architectures. From the linguistic perspective, our results suggest an alternative view of the relation between compositionality and language transmission-one in which the former might arise by chance or due to other factors, but then makes the resulting language much easier to be spread.

Compositionality and disentanglement Language is a way to represent meaning through discrete symbols. It is thus worth exploring the link between the area of language emergence and that of representation learning (Bengio et al., 2013). We took this route, borrowing ideas from research on disentangled representations to craft our compositionality measures. We focused in particular on the intuition that, if emergent languages must denote ensembles of primitive input elements, they are compositional when they use symbols to univocally denote input elements independently of each other.

While the new measures we proposed are not highly correlated with topographic similarity, in most of our experiments they did not behave significantly differently from the latter. On the one hand, given that topographic similarity is an established way to quantify compositionality, this serves as a sanity check on the new measures. On the other, we are disappointed that we did not find more significant differences between the three measures.

Interestingly one of the ways in which they did differ is that, when a language is positionally disentangled, (and, to a lesser extent, bag-of-symbols disentangled), it is very likely that the language will be able to generalize-a guarantee we don't have from less informative topographic similarity.

The representation learning literature is not only proposing disentanglement measures, but also ways to encourage emergence of disentanglement in learned representations. As we argued that compositionality has, after all, desirable properties, future work could adapt methods for learning disentangled representations (e.g., Higgins et al., 2017; Kim and Mnih, 2018) to let (more) compositional languages emerge.

\section{Acknowledgments}

We thank the reviewers for feedback that helped us to make the paper clearer.

\section{References}

Jacob Andreas. 2019. Measuring compositionality in representation learning. In Proceedings of ICLR.

Joris Baan, Jana Leible, Mitja Nikolaus, David Rau, Dennis Ulmer, Tim Baumgärtner, Dieuwke Hupkes, and Elia Bruni. 2019. On the realization of compositionality in neural networks. In Proceedings of $A C L$ BlackboxNLP Workshop.

Y. Bengio, A. Courville, and P. Vincent. 2013. Representation learning: A review and new perspectives. IEEE Transactions on Pattern Analysis and Machine Intelligence, 35(8).

Derek Bickerton. 2014. More than Nature Needs: Language, Mind, and Evolution. Harvard University Press, Cambridge, MA.

Alican Bozkurt, Babak Esmaeili, Dana H Brooks, Jennifer G Dy, and Jan-Willem van de Meent. 2019. Evaluating combinatorial generalization in variational autoencoders. arXiv preprint arXiv:1911.04594.

Philémon Brakel and Stefan Frank. 2009. Strong systematicity in sentence processing by simple recurrent networks. In Proceedings of CogSci.

Henry Brighton and Simon Kirby. 2006. Understanding linguistic evolution by visualizing the emergence of topographic mappings. Artificial life, 12(2):229242.

Rahma Chaabouni, Eugene Kharitonov, Emmanuel Dupoux, and Marco Baroni. 2019. Anti-efficient encoding in emergent communication. In Proceedings of NeurIPS.

Tian Qi Chen, Xuechen Li, Roger B Grosse, and David K Duvenaud. 2018. Isolating sources of disentanglement in variational autoencoders. In Proceedings of NeurIPS.

Kyunghyun Cho, Bart Van Merriënboer, Caglar Gulcehre, Dzmitry Bahdanau, Fethi Bougares, Holger Schwenk, and Yoshua Bengio. 2014. Learning phrase representations using rnn encoder-decoder for statistical machine translation. arXiv preprint arXiv: 1406.1078 .

Michael Cogswell, Jiasen Lu, Stefan Lee, Devi Parikh, and Dhruv Batra. 2019. Emergence of compositional language with deep generational transmission. arXiv preprint arXiv:1904.09067.

Hannah Cornish, Rick Dale, Simon Kirby, and Morten Christiansen. 2017. Sequence memory constraints give rise to language-like structure through iterated learning. PLoS ONE, 12(1):1-18.

Jerry Fodor and Ernest Lepore. 2002. The Compositionality Papers. Oxford University Press, Oxford, UK. 
Adele Goldberg. 2019. Explain Me This: Creativity, Competition, and the Partial Productivity of Constructions. Princeton University Press, Priinceton, NJ.

Shangmin Guo, Yi Ren, Serhii Havrylov, Stella Frank, Ivan Titov, and Kenny Smith. 2019. The emergence of compositional languages for numeric concepts through iterated learning in neural agents. arXiv preprint arXiv:1910.05291.

Irina Higgins, Loic Matthey, Arka Pal, Christopher Burgess, Xavier Glorot, Matthew Botvinick, Shakir Mohamed, and Alexander Lerchner. 2017. betaVAE: Learning basic visual concepts with a constrained variational framework. In Proceedings of ICLR

Dieuwke Hupkes, Verna Dankers, Mathijs Mul, and Elia Bruni. 2019. The compositionality of neural networks: integrating symbolism and connectionism. arXiv preprint arXiv:1908.08351.

James Hurford. 2014. The Origins of Language. Oxford University Press, Oxford, UK.

Eric Jang, Shixiang Gu, and Ben Poole. 2016. Categorical reparameterization with Gumbel-softmax. arXiv preprint arXiv:1611.01144.

Eugene Kharitonov and Marco Baroni. 2020. Emergent language generalization and acquisition speed are not tied to compositionality. arXiv preprint arXiv:2004.03420.

Eugene Kharitonov, Rahma Chaabouni, Diane Bouchacourt, and Marco Baroni. 2019. EGG: a toolkit for research on emergence of language in games. In Proceedings of EMNLP (System Demonstrations).

Hyunjik Kim and Andriy Mnih. 2018. Disentangling by factorising. arXiv preprint arXiv: 1802.05983.

Diederik P Kingma and Jimmy Ba. 2014. Adam: A method for stochastic optimization. arXiv preprint arXiv: 1412.6980

Simon Kirby. 2001. Spontaneous evolution of linguistic structure-an iterated learning model of the emergence of regularity and irregularity. IEEE Transactions on Evolutionary Computation, 5(2):102-110.

Simon Kirby, Monica Tamariz, Hannah Cornish, and Kenny Smith. 2015. Compression and communication in the cultural evolution of linguistic structure. Cognition, 141:87-102.

Satwik Kottur, José Moura, Stefan Lee, and Dhruv Batra. 2017. Natural language does not emerge 'naturally' in multi-agent dialog. In Proceedings of EMNLP.

Brenden Lake and Marco Baroni. 2018. Generalization without systematicity: On the compositional skills of sequence-to-sequence recurrent networks. In Proceedings of ICML.
Angeliki Lazaridou, Karl Moritz Hermann, Karl Tuyls, and Stephen Clark. 2018. Emergence of linguistic communication from referential games with symbolic and pixel input. In Proceedings of ICLR.

David Lewis. 1969. Convention. Harvard University Press, Cambridge, MA.

Fushan Li and Michael Bowling. 2019. Ease-ofteaching and language structure from emergent communication. arXiv preprint arXiv:1906.02403.

Francesco Locatello, Stefan Bauer, Mario Lucic, Gunnar Raetsch, Sylvain Gelly, Bernhard Schölkopf, and Olivier Bachem. 2019. Challenging common assumptions in the unsupervised learning of disentangled representations. In Proceedings of ICML.

Chris J Maddison, Andriy Mnih, and Yee Whye Teh. 2016. The concrete distribution: A continuous relaxation of discrete random variables. arXiv preprint arXiv:1611.00712.

Gary Marcus. 2003. The Algebraic Mind. MIT Press, Cambridge, MA.

Igor Mordatch and Pieter Abbeel. 2018. Emergence of grounded compositional language in multi-agent populations. In $A A A I$.

Ryan Nefdt. 2020. A puzzle concerning compositionality in machines. Mind and Machines. In press.

Martin Nowak and David Krakauer. 1999. The evolution of language. Proceedings of the National Academy of Sciences, 96(14):8028-8033.

Martin A Nowak, Joshua B Plotkin, and Vincent AA Jansen. 2000. The evolution of syntactic communication. Nature, 404(6777):495.

Peter Pagin and Dag Westerståhl. 2010. Compositionality II: Arguments and problems. Philosophy Compass, 5(3):265-282.

Barbara Partee. 2004. Compositionality in Formal Semantics. Blackwell, Malden, MA.

Steven Piantadosi, Harry Tily, and Edward Gibson. 2012. The communicative function of ambiguity in language. Cognition, 122(3):280-291.

Cinjon Resnick, Abhinav Gupta, Jakob Foerster, Andrew M Dai, and Kyunghyun Cho. 2019. Capacity, bandwidth, and compositionality in emergent language learning. arXiv preprint arXiv:1910.11424.

John Schulman, Nicolas Heess, Theophane Weber, and Pieter Abbeel. 2015. Gradient estimation using stochastic computation graphs. In Advances in $\mathrm{Neu}$ ral Information Processing Systems.

Raphael Suter, Djordje Miladinovic, Bernhard Schölkopf, and Stefan Bauer. 2019. Robustly disentangled causal mechanisms: Validating deep representations for interventional robustness. In Proceedings of ICML. 
Simon Townsend, Sabrina Engesser, Sabine Stoll, Klaus Zuberbühler, and Balthasar Bickel. 2018. Compositionality in animals and humans. PLOS Biology, 16(8):1-7.

Frank van der Velde, Gwendid van der Voort van der Kleij, and Marc de Kamps. 2004. Lack of combinatorial productivity in language processing with simple recurrent networks. Connection Science, 16(1):21-46.

Tessa Verhoef, Simon Kirby, and Bart de Boer. 2016. Iconicity and the emergence of combinatorial structure in language. Cognitive Science, 40(8):19691994.

Ronald J Williams. 1992. Simple statistical gradientfollowing algorithms for connectionist reinforcement learning. Machine learning, 8(3-4):229-256.

Shengjia Zhao, Hongyu Ren, Arianna Yuan, Jiaming Song, Noah Goodman, and Stefano Ermon. 2018. Bias and generalization in deep generative models: An empirical study. In Advances in Neural Information Processing Systems.

\section{Supplementary material}

\subsection{Grid search over $\left(i_{a t t}, i_{v a l}, c_{l e n}, c_{v o c}\right)$}

We report in Table 3 the different $\left(i_{a t t}, i_{v a l}, c_{l e n}\right.$, $\left.c_{v o c}\right)$ combinations we explored. They were picked according to the following criteria:

- $|C| \geq|I|$ so that agents are endowed with enough different messages to refer to all inputs;

- discard some $|C|>>|I|$ so that we have approximately the same number of settings $\operatorname{per}\left(i_{\text {att }}, i_{\text {val }}\right)$ (between 13 and 15 different $\left.\left(c_{v o c}, c_{l e n}\right)\right)$;

- include some $\left(c_{v o c}, c_{l e n}\right)$ that are large enough that they can be tested with all the considered $\left(i_{\text {att }}, i_{\text {val }}\right)$.

Unless it is mentioned explicitly, we run 10 different initializations per setting.

Table 3 shows that, for large $|I|$, GRU-agents need $|C|$ strictly larger than $|I|$. This suggests that, for large $|I|$, the emergence of a perfectly nonambiguous compositional languages, where each message symbol denotes only one attribute value and each value attribute is denoted by only one message symbol, is impossible.

\subsection{Behavior of the compositionality measures on hand-crafted miniature languages}

We construct 3 simple miniature languages to illustrate the different behaviors of topsim, posdis and bosdis: Lang1, Lang2 and Lang3. We fix $i_{\text {att }}=2$, $i_{v a l}=4, c_{l e n}=3$ and $c_{v o c}=8 .^{6}$ Table 4 shows the input-message mappings of each language and reports their degree of compositionality. Note that all languages respect a bijective mapping between inputs and messages.

Lang1 is perfectly posdis-compositional (posdis $=1$ ). However, topsim $<1$, as 2 symbols encode one attribute (we need the first two symbols to recover the value of the first attribute). Lang1 is penalized by topsim because it does not have a oneto-one attribute-position mapping; a feature that arguably is orthogonal to compositionality.

Lang2 and Lang3 are equally topsimcompositional. Nonetheless, they differ fundamentally in terms of the type of compositionality they feature. If Lang2 is more posdis-compositional, Lang3 is perfectly bosdis-compositional.

\subsection{Generalization for different agents' capacity}

We demonstrated in the main paper that agent's generalization correlates with input size. In fact, agents can successfully reconstruct new attribute combinations if trained on large input spaces. This could be due to agents overfitting when presented with few training samples. To test this hypothesis, we repeat the training/evaluation experiments with GRU agents of different capacities in the following settings: $\left(i_{\text {att }}=2, i_{\text {val }}=10\right)$, a small input space where agents do not generalize; and $\left(i_{a t t}=2\right.$, $i_{\text {val }}=100$ ), a large input space where agents generalize. ${ }^{7}$ Fig. 3 shows that, even for small-capacity agents (one-layer GRU with hidden state of size $100)$, test accuracy is 0 for $\left(i_{a t t}=2, i_{v a l}=10\right)$. Moreover, agents do not overfit when trained on $\left(i_{a t t}=2\right.$, $\left.i_{v a l}=100\right)$ even with two-layer GRUs with hidden state of size 500 .

\subsection{Input space density}

We showed in the main paper that generalization positively correlates with $|I|$. We further investigate here whether it is simply the increasing abso-

\footnotetext{
${ }^{6}$ Only Lang3 uses the whole available $c_{v o c}$

${ }^{7} \mathrm{We}$ only report experiments with GRUs, but the same results were replicated with differently-sized LSTMs.
} 


\begin{tabular}{|c|c|c|c|c|c|c|c|c|c|c|c|c|c|c|c|c|}
\hline$c_{v o c}$ & & & & 5 & & & & 10 & & & & 6 & & & & 00 \\
\hline$\left(i_{v a l}, i_{a t t}\right) \quad C_{l e n}$ & 2 & 3 & 4 & $\{6,8\}$ & 2 & 3 & 4 & $\{6,8\}$ & 2 & 3 & 4 & $\{6,8\}$ & 2 & 3 & 4 & $\{6,8\}$ \\
\hline$(4,4)$ & & & $\mathrm{X}$ & $\mathrm{X}$ & & $X$ & $\mathrm{X}$ & $\mathrm{X}$ & $X$ & & & $\mathrm{X}$ & $\mathrm{X}$ & & & $X$ \\
\hline$(5,2)$ & $X$ & $X$ & $\mathrm{X}$ & $\mathrm{X}$ & X & $X$ & $\mathrm{X}$ & $\mathrm{X}$ & $\mathrm{X}$ & & & $\mathrm{X}$ & $\mathrm{X}$ & & & $X$ \\
\hline$(5,3)$ & & $X$ & $X$ & $X$ & & $X$ & $X$ & $X$ & $X$ & & & $X$ & $X$ & & & $X$ \\
\hline$(5,4)$ & & & $\mathrm{X}$ & $\mathrm{X}$ & & $\mathrm{X}$ & $\mathrm{X}$ & $\mathrm{X}$ & $\mathrm{X}$ & & & $\mathrm{X}$ & $X$ & & & $X$ \\
\hline$(10,2)$ & & - & $\mathrm{X}$ & $\mathrm{X}$ & $X$ & $X$ & $\mathrm{X}$ & $X$ & $\mathrm{X}$ & & & $\mathrm{X}$ & $X$ & & & $X$ \\
\hline$(10,3)$ & & & & X & & - & $\mathrm{X}$ & $\mathrm{X}$ & $\mathrm{X}$ & $X$ & $\mathrm{X}$ & $\mathrm{X}$ & $\mathrm{X}$ & & & $X$ \\
\hline$(10,4)$ & & & & $\{-, X\}$ & & & - & $\mathrm{X}$ & & $X$ & $\mathrm{X}$ & $\mathrm{X}$ & - & $\mathrm{X}$ & $\mathrm{X}$ & $X$ \\
\hline$(16,2)$ & & & - & X & & $X$ & $X$ & $X$ & $X$ & & & $\mathrm{X}$ & $\mathrm{X}$ & & & $X$ \\
\hline$(25,2)$ & & & - & $\mathrm{X}$ & & - & $X$ & $\mathrm{X}$ & $\mathrm{X}$ & & & $\mathrm{X}$ & $X$ & & & $X$ \\
\hline$(50,2)$ & & & & $\mathrm{X}$ & & & - & $\mathrm{X}$ & - & $\mathrm{X}$ & $X$ & $\mathrm{X}$ & $X$ & $\mathrm{X}$ & $\mathrm{X}$ & $X$ \\
\hline$(100,2)$ & & & & $\{-, X\}$ & & & - & $X$ & & $\mathrm{X}$ & $\mathrm{X}$ & $X$ & - & $X$ & $X$ & $X$ \\
\hline
\end{tabular}

Table 3: Grid search. ' $\mathrm{X}$ ' indicates tested settings with at least one successful run. '-' indicates tested settings without any successful run. Finally, blank cells correspond to settings that were not explored for the reasons indicated in the text.

\begin{tabular}{lccc}
\hline Input & Lang1 & Lang2 & Lang3 \\
\hline 0,0 & $0,0,0$ & $0,0,0$ & $0,0,4$ \\
0,1 & $0,0,1$ & $0,0,1$ & $0,0,5$ \\
0,2 & $0,0,2$ & $0,0,2$ & $0,0,6$ \\
0,3 & $0,0,3$ & $0,0,3$ & $0,0,7$ \\
1,0 & $0,1,0$ & $1,2,0$ & $1,4,1$ \\
1,1 & $0,1,1$ & $1,2,1$ & $1,5,1$ \\
1,2 & $0,1,2$ & $1,2,2$ & $1,6,1$ \\
1,3 & $0,1,3$ & $1,2,3$ & $1,7,1$ \\
2,0 & $2,0,0$ & $2,3,0$ & $2,4,2$ \\
2,1 & $2,0,1$ & $2,3,1$ & $2,5,2$ \\
2,2 & $2,0,2$ & $2,3,2$ & $2,6,2$ \\
2,3 & $2,0,3$ & $2,3,3$ & $2,7,2$ \\
3,0 & $2,1,0$ & $3,1,0$ & $3,4,3$ \\
3,1 & $2,1,1$ & $3,1,1$ & $3,3,5$ \\
3,2 & $2,1,2$ & $3,2,1$ & $3,3,6$ \\
3,3 & $2,1,3$ & $3,3,1$ & $3,3,7$ \\
\hline \hline topsim & 0.82 & 0.75 & 0.75 \\
posdis & 1 & 0.79 & 0.43 \\
bosdis & 0.42 & 0.13 & 1 \\
\hline
\end{tabular}

Table 4: Input-message mappings and compositionality measures for the miniature languages.

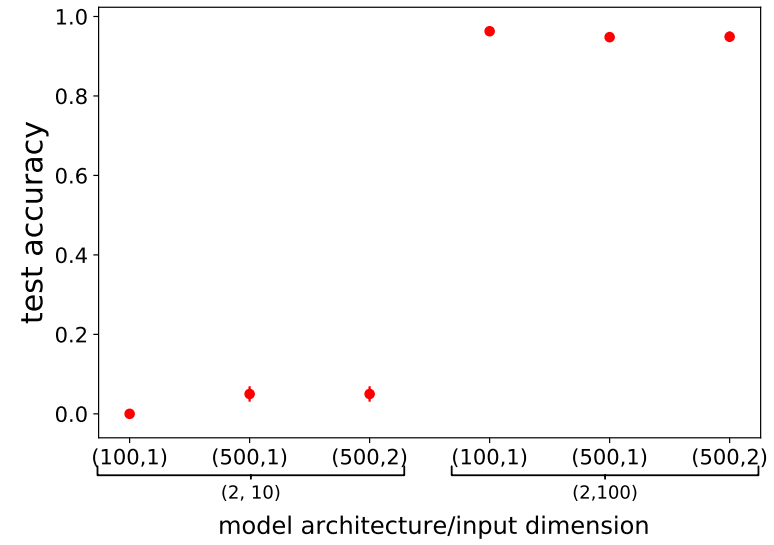

Figure 3: Average accuracy on unseen combinations as a function of agents capacity ((hidden size, number of layers)) for input sizes $\left(i_{a t t}=2, i_{v a l}=10\right)$ and $\left(i_{a t t}=2, i_{\text {val }}=100\right)$. Vertical bars represent SEM.

lute number of distinct training samples that is at the root of this phenomenon, or whether the variety of seen inputs also plays a role, independently of absolute input size.

To verify this, we design an experiment where we keep the absolute number of distinct input samples constant, but we change their density, defined as the proportion of sampled items over the the size of the space they are sampled from. When sampling points from a small space, on average each value of an attribute will occur with a larger range of values from other attributes, compared to a larger space, which might provide more evidence about the combinatorial nature of the underlying space. 
In practice, we fix $\left(c_{l e n}=3, c_{v o c}=100, i_{a t t}=2\right)$ and sample 10000 points from spaces with $i_{v a l}=100$ (density=1), $i_{\text {val }}=140$ (density=0.51) and $i_{\text {val }}=200$ (density $=0.25$ ), respectively. As usual, we use $90 \%$ of the data for training, $10 \%$ for testing. In all cases, we make sure that all values are seen at least once during training (as visually illustrated in Fig. 4).

We obtain test accuracies of $92.7 \%, 66.7 \%$ and $22.8 \%$ for densities $1,0.51$ and 0.25 respectively. That is, the high generalization observed in the main paper is (also) a consequence of density, and hence combinatorial variety, of the inputs the agents are trained on, and not (only) of the number of training examples.

\subsection{Impact of channel capacity on generalization}

We showed in the main paper that generalization is very sensitive to input size. In this section, we focus on the relation between channel capacity $|C|$ and generalization.

First, when we aggregate across input sizes, Fig. 5 shows that $|C|$ has a just small effect on generalization, with a low Spearman correlation $\rho=0.14$. Next, if we study this relation for specific large $|I|$ (where we observe generalization), we notice in Fig. 6 that agents need to be endowed with a $|C|$ above a certain threshold, with $\frac{|C|}{|I|}>1$, in order to achieve almost perfect generalization. Moreover, contradicting previous claims (e.g., Kottur et al., 2017), having $|C|>>|I|$ does not harm generalization.

\subsection{Impact of channel capacity on the compositionality measures}

A good compositionality measure should describe the structure of the language independently of the used channel, so the corresponding score should not be greatly affected by $|C|$. However, Fig. 7 shows clear negative correlations of both topsim and bosdis with $|C|$.

\subsection{Analysis of example medium- and low-posdis languages}

We present more data about the medium-posdis language analyzed in the main article, and we provide comparable evidence for a language with similarly excellent generalization $(>99 \%)$ but very low posdis (0.05), that we will call here low-posdis. The latter language is depicted in black in Fig. 2 of the main text. Both languages come from the training configuration with 2 100-valued input attributes and 3 100-symbol positions.

Mutual information profiles. Table 5 reports mutual information for the two languages. Note how the highly entangled low-posdis is almost uniform across the table cells.

\begin{tabular}{l|ll|ll}
\multicolumn{1}{c}{} & \multicolumn{2}{c}{ medium-posdis } & \multicolumn{2}{c}{ low-posdis } \\
& att1 & att2 & att1 & att2 \\
\hline pos1 & 1.10 & 2.01 & 1.72 & 1.95 \\
pos2 & 0.19 & 4.16 & 1.74 & 1.71 \\
pos3 & 4.44 & 0.13 & 2.16 & 1.77
\end{tabular}

Table 5: Mutual information of each position with each attribute for the studied languages.

Vocabulary usage. Considering all messages produced after training for the full training and test set inputs, effective vocabulary usage for pos 1 , pos 2 and pos 3 are as follows (recall that 100 symbols are maximally available):

- medium-posdis: 91, 96, 98

- low-posdis: 99, 99, 100

Although vocabulary usage is high in both cases, medium-posdis is slightly more parsimonious than low-posdis.

Ablation studies. Table 6 reports ablation experiments with both languages. The results for medium-posdis are discussed in the main text. We observe here how virtually any ablation strongly impacts accuracy in denoting either attribute by the highly entangled low-posdis language. This points to another possible advantage of (partially) disentangled languages such as medium-posdis: since pos 2 and $p o s 3$ are referring to att 2 and att 1 independently, in ablations in which they are untouched, Receiver can still retrieve partial information, by often successfully guessing the attribute they each refer to. We also report in the table the effect of shuffling across the positions of each message. This is very damaging not only for medium-posdis, but for low-posdis as well, showing that even the latter is exploiting positional information, albeit in an inscrutable, highly entangled way. Note in Fig. 2 of the main article that neither language has high bos. 


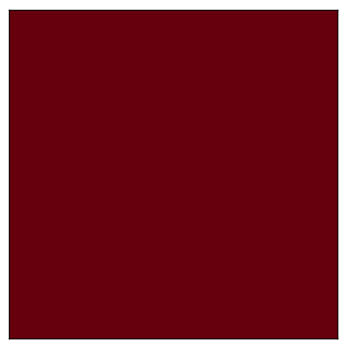

(a) density $=1$

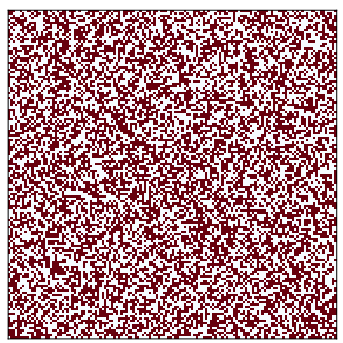

(b) density $=0.51$

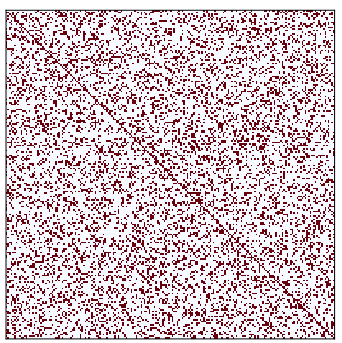

(c) density $=0.25$

Figure 4: Sampling the same number of input instances $(=10000)$ with different densities. The axes of the shown matrices represent the values of two attributes, with the dark-red cells standing for inputs that were sampled. We ensure that each value of each attribute is picked at least once by always sampling the full diagonal.

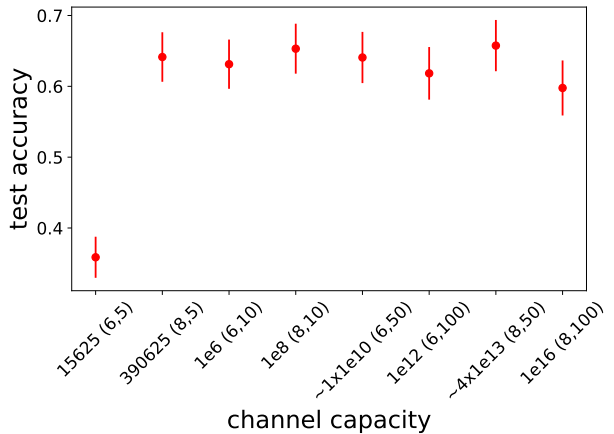

Figure 5: Average accuracy on unseen combinations as a function of channel capacity of the successful runs. The $\mathrm{x}$-axis is ordered by increasing channel capacity. In the brackets we note $\left(c_{l e n}, c_{v o c}\right)$. Vertical bars represent SEM.

\subsection{Effect of channel capacity on ease of transmission}

Table 7 replicates the ease-of-transmission analysis presented in the main text across various channel capacities. We observe in most cases a significantly positive correlation, that is even higher (1) for larger Receivers and (2) for emergent languages with shorter messages ( $\operatorname{smaller} c_{l e n}$ ).

\begin{tabular}{rr|r|r|r|r|r|r}
\multicolumn{1}{c}{} & \multicolumn{3}{c}{ medium-posdis low-posdis } \\
& & att 1 & att 2 & both & att1 & att2 & both \\
\hline fixing pos1 & 1 & 3 & 0 & 4 & 5 & 0 \\
1 position pos2 & 1 & 68 & 0 & 4 & 4 & 0 \\
pos3 & 89 & 1 & 1 & 8 & 5 & 0 \\
\hline shuffling pos1 & 89 & 69 & 61 & 31 & 18 & 6 \\
1 position pos 2 & 100 & 3 & 3 & 30 & 25 & 8 \\
pos3 & 1 & 100 & 1 & 15 & 20 & 3 \\
\hline shuffling msg & 1 & 2 & 0 & 2 & 4 & 0
\end{tabular}

Table 6: Feeding shuffled messages from the mediumposdis and low-posdis languages to the corresponding trained Receivers. Mean percentage accuracy across 10 random shufflings (standard deviation is always $\approx 0$ ) when: top: symbols in all positions but one are shuffled across the data-set; middle: symbols in a single position are shuffled across the data-set; bottom: shuffling the symbols within each message (ensuring all symbols move). The data-set includes all training and test messages produced by the trained Sender and correctly decoded in their original form by Receiver ( $>99 \%$ of total messages). 


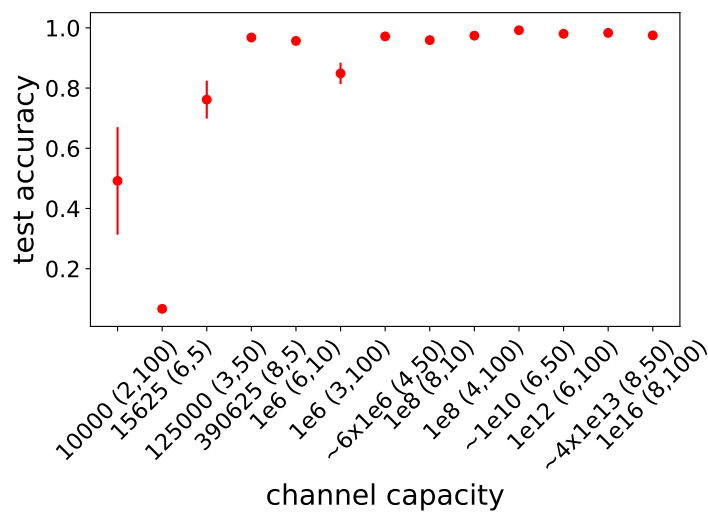

(a) $\left(i_{\text {att }}=2, i_{\text {val }}=50\right)$

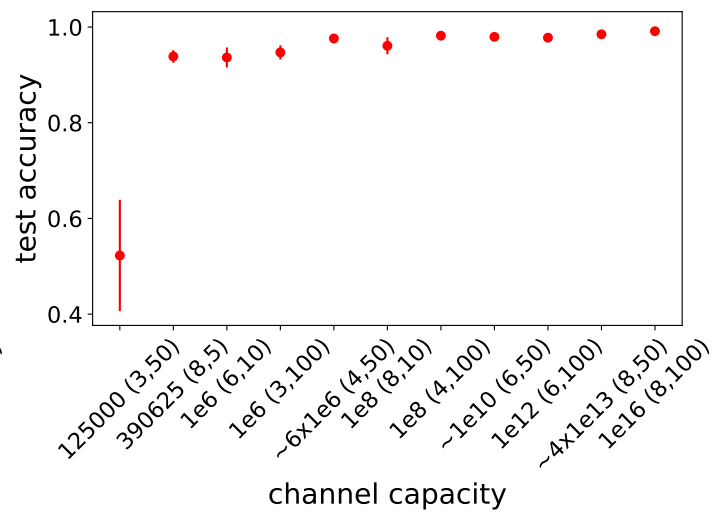

(b) $\left(i_{\text {att }}=2, i_{\text {val }}=100\right)$

Figure 6: Average accuracy on unseen combinations as a function of channel capacity of the successful runs for two different $\left(i_{a t t}, i_{v a l}\right)$. The $\mathrm{x}$-axis is ordered by increasing channel capacity. In the brackets we note $\left(c_{l e n}, c_{v o c}\right)$. Vertical bars represent SEM.

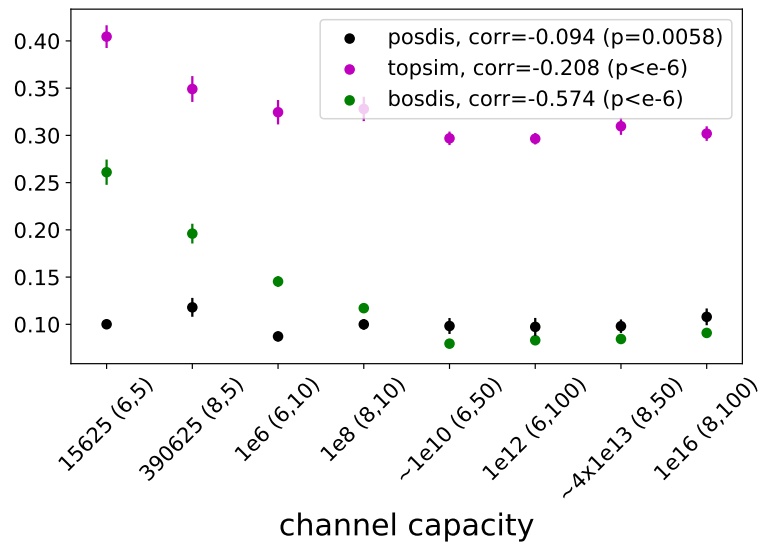

Figure 7: Average of different compositionality measures in function of channel capacity $\left.\left(c_{v o c}, c_{l e n}\right)\right)$. Vertical bars represent SEM. 


\begin{tabular}{|c|c|c|c|c|c|c|c|c|c|c|}
\hline \multirow[b]{2}{*}{$\left(c_{l e n}, c_{v o c}\right)$} & \multirow[b]{2}{*}{ measure } & \multicolumn{3}{|c|}{ posdis } & \multicolumn{3}{|c|}{ bosdis } & \multicolumn{3}{|c|}{ topsim } \\
\hline & & $\begin{array}{l}\text { GRU } \\
(500)\end{array}$ & $\begin{array}{l}\text { GRU } \\
(50)\end{array}$ & $\begin{array}{l}\text { FFN } \\
(500)\end{array}$ & $\begin{array}{l}\text { GRU } \\
(500)\end{array}$ & $\begin{array}{l}\text { GRU } \\
(50)\end{array}$ & $\begin{array}{l}\text { FFN } \\
(500)\end{array}$ & $\begin{array}{l}\text { GRU } \\
(500)\end{array}$ & $\begin{array}{l}\text { GRU } \\
(50)\end{array}$ & $\begin{array}{l}\text { FFN } \\
(500)\end{array}$ \\
\hline \multirow{2}{*}{$(3,50)$} & Learning Speed & 0.82 & 0.78 & 0.74 & 0.71 & 0.67 & 0.62 & 0.72 & 0.74 & 0.66 \\
\hline & Generalization & 0.77 & 0.77 & 0.75 & 0.61 & 0.62 & 0.66 & 0.75 & 0.76 & 0.74 \\
\hline \multirow{2}{*}{$(4,50)$} & Learning Speed & 0.79 & 0.44 & 0.48 & 0.76 & 0.51 & 0.47 & 0.89 & 0.59 & 0.61 \\
\hline & Generalization & 0.73 & - & 0.50 & 0.77 & 0.27 & 0.54 & 0.84 & 0.41 & 0.61 \\
\hline \multirow{2}{*}{$(6,50)$} & Learning Speed & 0.82 & 0.77 & 0.79 & 0.79 & 0.76 & 0.77 & 0.89 & 0.85 & 0.87 \\
\hline & Generalization & 0.78 & 0.56 & 0.69 & 0.76 & 0.55 & 0.67 & 0.85 & 0.65 & 0.77 \\
\hline \multirow{2}{*}{$(8,50)$} & Learning Speed & 0.75 & 0.56 & 0.78 & 0.80 & 0.68 & 0.78 & 0.75 & 0.55 & 0.71 \\
\hline & Generalization & 0.67 & 0.27 & 0.68 & 0.78 & 0.41 & 0.70 & 0.53 & - & 0.54 \\
\hline \multirow{2}{*}{$(10,50)$} & Learning Speed & 0.51 & 0.29 & 0.60 & 0.42 & 0.31 & 0.48 & 0.72 & 0.49 & 0.73 \\
\hline & Generalization & 0.39 & - & 0.44 & 0.47 & - & 0.36 & 0.41 & 0.27 & 0.57 \\
\hline \multirow{2}{*}{$(12,50)$} & Learning Speed & - & - & - & 0.33 & - & - & 0.49 & - & 0.35 \\
\hline & Generalization & - & -0.28 & - & - & - & - & - & - & - \\
\hline \multirow{2}{*}{$(3,100)$} & Learning Speed & 0.87 & 0.71 & 0.35 & 0.85 & 0.68 & 0.33 & 0.87 & 0.71 & 0.35 \\
\hline & Generalization & 0.80 & 0.55 & 0.50 & 0.81 & 0.55 & 0.51 & 0.79 & 0.54 & 0.48 \\
\hline \multirow{2}{*}{$(4,100)$} & Learning Speed & 0.84 & 0.54 & 0.43 & 0.82 & 0.54 & 0.46 & 0.86 & 0.57 & 0.49 \\
\hline & Generalization & 0.82 & 0.38 & 0.47 & 0.80 & 0.39 & 0.47 & 0.82 & 0.41 & 0.48 \\
\hline \multirow{2}{*}{$(6,100)$} & Learning Speed & 0.88 & 0.83 & 0.80 & 0.89 & 0.78 & 0.78 & 0.94 & 0.87 & 0.83 \\
\hline & Generalization & 0.87 & 0.68 & 0.68 & 0.90 & 0.69 & 0.67 & 0.90 & 0.70 & 0.68 \\
\hline \multirow{2}{*}{$(10,100)$} & Learning Speed & 0.85 & 0.58 & 0.62 & 0.82 & 0.59 & 0.64 & 0.72 & 0.74 & 0.66 \\
\hline & Generalization & 0.86 & 0.39 & 0.47 & 0.81 & 0.50 & 0.37 & 0.72 & 0.35 & 0.46 \\
\hline \multirow{2}{*}{$(8,100)$} & Learning Speed & 0.73 & 0.58 & 0.65 & 0.79 & 0.59 & 0.65 & 0.70 & 0.57 & 0.66 \\
\hline & Generalization & 0.69 & 0.39 & 0.37 & 0.67 & 0.37 & 0.37 & 0.49 & 0.34 & 0.46 \\
\hline \multirow{2}{*}{$(12,100)$} & Learning Speed & 0.39 & - & 0.27 & 0.69 & - & 0.40 & 0.67 & - & 0.51 \\
\hline & Generalization & 0.38 & - & 0.34 & 0.52 & - & 0.38 & 0.36 & - & - \\
\hline \multirow{2}{*}{ Average } & Learning Speed & 0.75 & 0.62 & 0.61 & 0.72 & 0.63 & 0.59 & 0.79 & 0.67 & 0.62 \\
\hline & Generalization & 0.71 & 0.42 & 0.54 & 0.72 & 0.49 & 0.51 & 0.70 & 0.51 & 0.63 \\
\hline
\end{tabular}

Table 7: Statistically significant $(p<0.01)$ Spearman correlations between retraining performance (measured by new Receiver Learning Speed and Generalization) and compositionality measures (posdis, bosdis and topsim) for $\left(i_{a t t}=2, i_{v a l}=100\right)$ and different channel capacity. '-' indicates no significant correlations. 